$\Rightarrow$ MILESTONE 17

\section{Dilute for impact}

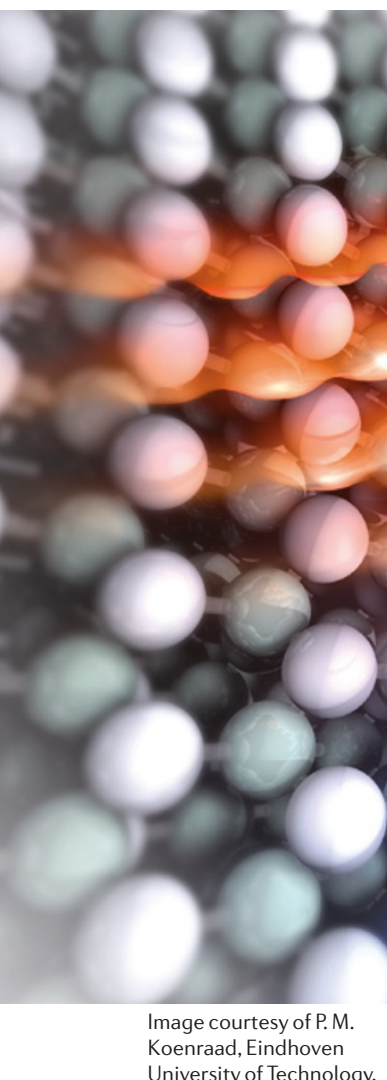

could be probed. At the same time, pronounced magnetic properties could be observed — such as the spin splitting of electronic or impurity bands.

A further breakthrough came in the early 1990s, with the advent of low-temperature molecular-beam epitaxy. By growing semiconductor films under conditions that were far from thermal equilibrium, it became possible to introduce manganese impurities into III-V materials (which is more difficult under equilibrium conditions owing to the low solubility of manganese). Hideo Ohno and colleagues then demonstrated, in 1992, ferromagnetic order in the DMS (In,Mn)As — indium arsenide containing only $1.3 \%$ manganese - by measuring magneto-transport and, in particular, an anomalous Hall effect in the material. The work was followed up, in 1996, with proof of ferromagnetism in doped gallium arsenide - (Ga,Mn)As - at temperatures up to $110 \mathrm{~K}$. As GaAs can be used in electronic devices that operate at room temperature, these studies established the basis for research into 'technologically relevant' DMSs.

The 1990s also brought theoretical work by Tomasz Dietl, in collaboration with Ohno's group, which explained the origin of ferromagnetism in ( $\mathrm{Ga}, \mathrm{Mn})$ As using a model developed, by Clarence Zener in 1950, for ferromagnetism in transition metals. According to this model, the magnetic order originates from the delocalized holes that mediate the interaction between localized magnetic moments. The importance of the work was twofold. First, the carrier-mediated magnetic order suggested the possibility of controlling the ferromagnetism using electric fields - which was soon demonstrated - and, beyond that, the development of efficient spintronics devices. Second, the Dietl model provided an effective recipe for calculating the Curie temperature of other zinc-blende and wurzite semiconductors, to advance the search for a room-temperature DMS. In particular, Dietl showed that DMSs based on zinc oxide ( $\mathrm{ZnO}$ ) or gallium nitride $(\mathrm{GaN})$ could have Curie temperatures as high as $300 \mathrm{~K}$.

Investigations since then have indeed revealed room-temperature ferromagnetism in oxides and semiconductors that include $\mathrm{ZnO}$ or $\mathrm{GaN}$. However, it is yet to be proved that the carrier-mediated mechanism proposed by Dietl is really at work in these systems. The origin of this ferromagnetism - and its potential use in spintronics devices - is still a matter of controversy, yet the search for a carrier-mediated room-temperature DMS continues.

Fabio Pulizzi, Associate Editor, Nature Materials

ORIGINAL RESEARCH PAPERS Von Molnar, S. \& Methfessel, S. Giant negative magnetoresistance in ferromagnetic Eu1-xGdxSe.J. Appl. Phys. 38, 959-964 (1967)| Gałązka, R. R. in Proceedings of the 14th International Conference on the Physics of Semiconductors (ed. Wilson, B. L. H.) 133 (Institute of Physics, Bristol, 1978) | Munekata, H. et al. Diluted magnetic III-V semiconductors. Phys. Rev. Lett. 63, 1849-1852 (1989) |Ohno, H., Munekata, H., Penny, T., von Molnár, S. \& Chang, L. L. Magnetotransport properties of p-type (In,Mn)As diluted magnetic III-IV semiconductors. Phys. Rev. Lett. 68, 2664-2667 (1992) | Ohno, H. et al. (Ga,Mn)As: a new ferromagnetic semiconductor based on GaAs Appl. Phys. Lett. 69, 363-365 (1996) | Dietl, T., Ohno, H., Matsukura, F., Cibert, J. \& Ferrand, D. Zener model description of ferromagnetism in zinc-blende magnetic semiconductors. Science 287, 1019-1022 (2000) |Ohno, H. et al. Electricfield control of ferromagnetism. Nature 408 , 944-946 (2000)

FURTHER READING Furdyna, J. K. \& Kossut, J. (eds) Diluted Magnetic Semiconductors, Semiconductors and Semimetals Vol. 25 (Academic, London, 1988) 